\title{
Superlens-cloaking of small dielectric bodies in the quasistatic regime
}

\author{
Oscar P. Bruno ${ }^{\text {a) }}$ and Stéphane Lintner \\ Applied and Computational Mathematics, Caltech, Pasadena, California 91125, USA
}

(Received 24 August 2007; accepted 22 October 2007; published online 26 December 2007)

\begin{abstract}
It has been conjectured that, in the quasistatic regime, dielectric bodies of finite size could be perfectly cloaked by certain cylindrical arrangements of materials of positive and negative permittivities known as superlenses. We show that, although they do not cloak perfectly dielectrics objects of any size, cylindrical superlenses do cloak, to a significant extent, dielectric bodies of small size. (C) 2007 American Institute of Physics. [DOI: 10.1063/1.2821759]
\end{abstract}

Recent advances in material sciences have made it possible to construct materials whose effective electrical permittivities and magnetic permeabilities are negative over various ranges of frequencies. ${ }^{1}$ As shown in Refs. 2 and 3, such materials could be used for the design of cloaking devices: at a given frequency, a spherical volume could be cloaked by means of a spherical shell within which the electric permittivity and magnetic permeability vary in certain prescribed ways. At the given frequency, any object contained within the spherical volume would be invisible to outside observers.

A different cloaking device, the "cylindrical superlens," was proposed in Ref. 4. This device consists of a cylindrical core $r<r_{c}$ containing material of permittivity $\varepsilon_{c}=1$ (where $r$ denotes the distance to the cylinder center), surrounded by a cylindrical shell $r_{c}<r<r_{s}$ containing a material of permittivity $\varepsilon_{s}=-1+i \delta, \delta>0$. The medium in the region $r>r_{s}$ is assumed to have permittivity $\varepsilon_{m}=1$. As shown in Ref. 4, in the small loss $(\delta \rightarrow 0)$ and quasistatic (zero-frequency) limits, the superlens possesses the following two properties: (1) it is invisible (it does not cause modifications in any external quasistatic field) and (2) it creates a dipole cloaking region around itself: placing any finite number of polarizable (line) dipoles in the region $r_{s}<r<r_{\#}=\sqrt{r_{s}^{3} / r_{c}}$ does not give rise to changes on the total field at distances larger than $r_{\# \text {. Cylin- }}$ drical superlenses thus present an interesting alternative to the cloaking structures introduced in Ref. 1: they induce cloaking regions that lie outside the device itself.

In view of the dipole-cloaking properties of cylindrical superlenses, and since dielectric materials can be viewed as arrays of polarizable dipoles, it is reasonable to suggest, as was done in Ref. 4, that a cylindrical dielectric body placed within the cloaking region of a superlens may be cloaked perfectly. In this contribution we show that, while such a perfect-cloaking conjecture is not strictly valid, cylindrical superlenses give rise nonetheless to significant dielectriccloaking properties for small dielectric cylinders.

Our analysis relies on the use of a highly accurate numerical method based on integral equations and a corresponding computational implementation capable of dealing with the numerically challenging $\delta \rightarrow 0$ limit. Our numerical method is applicable to cylindrical configurations of arbitrary cross section as depicted in Fig. 1: we assume that the core

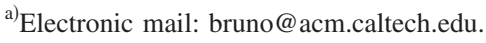

and shell are delimited by curves $\Gamma_{c}$ and $\Gamma_{s}$, respectively, and we allow for one or more dielectric cylindrical scatterers, with boundary $\Gamma_{0}$, lying somewhere outside the shell; in absence of such additional dielectric scatterers we simply set $\Gamma_{0}=\varnothing$. We assume that all the curves are smooth with outer normals $n=n(\mathbf{r})$, we denote by $\varepsilon_{c}, \varepsilon_{s}$, and $\varepsilon_{0}$ as the permittivities in the core, shell, and scatterer, respectively, and we let $\varepsilon(\mathbf{r})$ denote the dielectric permittivity at point $\mathbf{r}=(x, y)$.

To address issues brought up in Ref. 4 we also consider configurations such as that depicted in Fig. 1, but which, instead of a dielectric scatterer, include a finite number of polarizable (line) dipoles outside $\Gamma_{s}$. The field scattered by an array of polarizable dipoles located at points $\left(x_{j}, y_{j}\right), j$ $=1, \ldots, N$, is given by

$$
u^{\operatorname{dip}}(x, y)=\sum_{j=1}^{N} \mathbf{P}^{j} \cdot \nabla \log \sqrt{\left(x-x_{j}\right)^{2}+\left(y-y_{j}\right)^{2}},
$$

where denoting by $E^{r}=\nabla u_{i}^{r}$ the field generated by all sources except the "self-term" $i$ th dipole itself $\left\{E^{r}\right.$ thus includes the incident field $\nabla u^{\text {inc }}$, the field $\sum_{j \neq i}^{N} \mathbf{P}^{j}\left[\nabla \log \sqrt{\left(x-x_{j}^{d}\right)^{2}+\left(y-y_{j}^{d}\right)^{2}}\right]$ scattered by all other dipoles and the field $\nabla u^{\text {diel }}$ scattered by all dielectric bodies the moment $\mathbf{P}^{i}$ of the $i$ th dipole is given by

$$
\mathbf{P}^{i}=\alpha_{i} \nabla u_{i}^{r}\left(x_{i}, y_{i}\right)
$$

In absence of polarizable dipoles we simply set $N=0$.

The overall scattering problems we consider can then be stated as follows: given an incident field $E^{\text {inc }}=\nabla u^{\text {inc }}$ - where $u^{\text {inc }}=u^{\text {inc }}(x, y)$ is a harmonic function, $\nabla^{2} u^{\text {inc }}=0$, in a region containing the cylindrical structure,-we seek the overall scattered field $E^{\text {scat }}=\nabla u^{\text {scat }}$, where $u^{\text {scat }}=u^{\text {diel }}+u^{\text {dip }}$. The potential $u^{\text {diel }}$ is harmonic everywhere except at the curves $\Gamma_{c}$, $\Gamma_{s}$, and $\Gamma_{0}$, the potential $u^{\text {dip }}$ is given by Eq. (1) with moments satisfying Eq. (2), and, in addition: (i) $u^{\text {diel }}$ is continuous throughout $\mathbb{R}^{2}$; (ii) denoting by $u^{\text {tot }}=u^{\text {inc }}+u^{\text {scat }}$ the potential associated with the total field, the quantity $\varepsilon(\mathbf{r}) \partial u^{\text {tot }} / \partial n(\mathbf{r})$ is continuous across $\Gamma_{c}, \Gamma_{s}$, and $\Gamma_{0}$; and (iii) $u^{\text {diel }}$ is bounded as $r \rightarrow \infty$.

We will obtain the potential $u^{\text {diel }}$ in the form of a single layer potential arising from a density on each curve, 


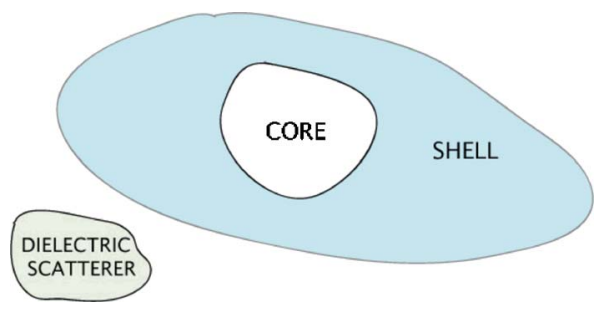

FIG. 1. (Color online) Cylindrical configuration: core, shell, and dielectric scatterer.

$$
u^{\text {diel }}=S_{\Gamma_{s}}\left(\varphi_{s}\right)+S_{\Gamma_{c}}\left(\varphi_{c}\right)+S_{\Gamma_{0}}\left(\varphi_{0}\right),
$$

where for a curve $\Gamma$ and a function $\varphi$ defined on $\Gamma, S_{\Gamma}(\varphi)$ is given by

$$
S_{\Gamma}(\varphi)(\xi)=\frac{1}{2 \pi} \int_{\Gamma} \log \left|\xi-\xi^{\prime}\right| \varphi\left(\xi^{\prime}\right) d l_{\xi^{\prime}}, \quad \xi \in \Gamma .
$$

The properties of single layer potentials (4) are well known: ${ }^{5}$ defining

$$
K_{\Gamma}^{\prime}(\varphi)(\xi)=\frac{1}{2 \pi} \int_{\Gamma} \frac{\partial \log \left|\xi-\xi^{\prime}\right|}{\partial n_{\xi}} \varphi\left(\xi^{\prime}\right) d l \xi^{\prime}, \quad \xi \in \Gamma,
$$

in view of earlier prescriptions on the potentials $u^{\mathrm{diel}}$ and $u^{\mathrm{dip}}$ and denoting $u^{i+d}=u^{\text {inc }}+u^{\text {dip }}$, it follows that $u^{\text {scat }}=u^{\text {diel }}+u^{\text {dip }}$ is the solution to the superlens scattering problem provided the integral equations

$$
(S)\left\{\begin{array}{l}
\eta_{s c} \frac{\varphi_{c}}{2}+K_{c}^{\prime}\left(\varphi_{c}\right)=-\frac{\partial}{\partial n_{c}}\left[u^{i+d}+S_{\Gamma_{s}}\left(\varphi_{s}\right)+S_{\Gamma_{0}}\left(\varphi_{0}\right)\right] \\
\eta_{m s} \frac{\varphi_{s}}{2}+K_{s}^{\prime}\left(\varphi_{s}\right)=-\frac{\partial}{\partial n_{s}}\left[u^{i+d}+S_{\Gamma_{c}}\left(\varphi_{c}\right)+S_{\Gamma_{0}}\left(\varphi_{0}\right)\right] \\
\eta_{m 0} \frac{\varphi_{0}}{2}+K_{c}^{\prime}\left(\varphi_{0}\right)=-\frac{\partial}{\partial n_{0}}\left[u^{i+d}+S_{\Gamma_{c}}\left(\varphi_{c}\right)+S_{\Gamma_{s}}\left(\varphi_{s}\right)\right]
\end{array}\right.
$$

are satisfied on $\Gamma_{c}, \Gamma_{s}$, and $\Gamma_{0}$, respectively, and provided Eqs. (1) and (2) hold. Here we set

$$
\begin{aligned}
& \eta_{s c}=\frac{\varepsilon_{c}+\varepsilon_{s}}{\varepsilon_{s}-\varepsilon_{c}}, \\
& \eta_{m s}=\frac{\varepsilon_{s}+\varepsilon_{m}}{\varepsilon_{m}-\varepsilon_{s}}, \\
& \eta_{m 0}=\frac{\varepsilon_{0}+\varepsilon_{m}}{\varepsilon_{m}-\varepsilon_{0}} .
\end{aligned}
$$

The integral operators involved in the system of equations $(S)$ have analytic kernels and are, in particular, compact. For nonvanishing values of $\eta_{c s}, \eta_{m s}$, and $\eta_{m 0}$ and for an arbitrary function $u^{i+d}$ the system $(S)$ constitutes a Fredholm equation of the second kind, and thus, in view of the Fredholm theory the system is uniquely solvable except for a discrete set of values of $\eta_{c s}, \eta_{m s}$, and $\eta_{m 0}$. The special case of a perfect superlens $(\delta=0)$ does not fall under this scope, however, since for a perfect superlens both $\eta_{c s}$ and $\eta_{m s}$ vanish. The two first equations in $(S)$ become first kind integral equations and $(S)$ may not admit solutions. In fact, it was already established in Ref. 2 that the response of the circular superlens to an illuminating dipole could become unphysical if the dipole lies sufficiently close to the superlens: for such cases a solution of our problem does not exist. A more general discussion can be given in the framework of the present integral-equation formulation: since all the kernels involved are analytic, the singular values of the associated operators decay exponentially fast, ${ }^{5}$ and only for right-hand sides whose singular-vector expansion coefficients tend to zero even faster can solutions exist. To have a solvable problem it is thus generally necessary to allow for losses in the materials: $\varepsilon_{s}=-1+i \delta, \delta>0$. For any loss value $\delta>0$ the Fredholm theory applies to $(S)$; in particular, since the spectrum of our problem is discrete, there is a range of values $0<\delta<\delta *$ for which $(S)$ is uniquely solvable for an arbitrary right-hand side. This discussion, finally, extends directly to the case in which $N>0$ (dipoles are present) and Eqs. (1) and (2) are enforced: the complete $2 N \times 2 N$ matrix associated with the dipole distribution is invertible for generic spatial distributions $\left(x_{j}, y_{j}\right), j=1, \ldots, N$.

To solve our equations in either the cases $N>0$ or $N$ $=0, \Gamma_{0}=\varnothing$, or $\Gamma_{0} \neq \varnothing$, we assume that each curve $\Gamma$ is described by a parametrization $\xi(t)=[x(t), y(t)](t \in[0,2 \pi])$, and we solve for the values of $\varphi[\xi(t)]$ at $M$ points in each curve by inverting a discrete approximation of $(S)$ together with Eqs. (1) and (2). For the analytic and periodic kernels under consideration, the trapezoidal integration rule gives rise to exponentially fast convergence of the numerical solutions as the discretizations are refined; see, e.g., Ref. 5. As noted in Ref. 2, for values of $\delta$ decreasing towards zero, the corresponding solutions of our equations become increasingly oscillatory; in the present context, as $\delta$ decreases our numerical algorithm requires larger and larger values of $M$ to yield a given accuracy. As a preliminary test of correctness of our formulation, however, we verified that our solver reproduces all of the specific solution values provided in Ref. 4 (see the captions of Figs. 1, 2, and 3 in that paper).

An issue that requires attention is the conditioning of the linear algebra problem as $\delta$ approaches zero: for small values of $\delta$ the condition number of the discretized versions of the system $(S)$ is in practice found to be roughly of the order of $1 / \delta$. Thus, the numerical inversion of $(S)$ can become quite a delicate matter: for values of the order of $\delta=10^{-12}$, which are used in Ref. 4, limited accuracy results. As it turns out, for theoretical reasons, some interesting cases in our experiments require values of $\delta$ as small as $10^{-20}$ : even though unphysical, consideration of solutions for such values of $\delta$ give rise to useful insights into the limiting process $\delta \rightarrow 0$. For this reason, our solver was implemented in $C^{++}$so as to take advantage of the "qd.h" library, ${ }^{6}$ and thus producing solutions without difficulty in triple or even quadruple precision. The resulting solver is very powerful: for the most challenging case considered in this paper (the smallest scatterer in the $\delta=10^{-20}$ curve in Fig. 2), this solver produces many correct significant figures using small numbers $(M \sim 900)$ of discretization points (unknowns) in a computing time of the order of two minutes in a single Intel Xeon processor at 3.00 GHz.

In what follows, we report on the results of a variety of numerical experiments we conducted for superlens configu- 


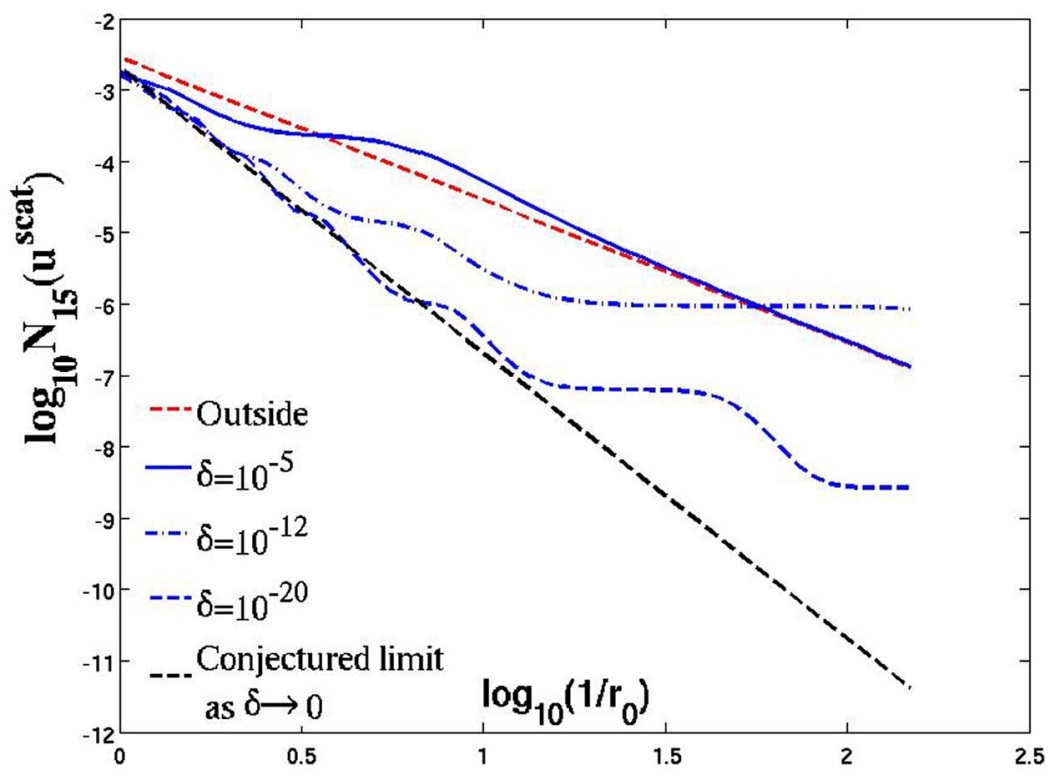

FIG. 2. (Color online) Cloaking of a dielectric body by a circular superlens as a function of the dielectric's size for various values of $\delta$. rations, involving superlenses of circular and elliptical cross sections, by means of the numerical methods described earlier. In all cases we use incident fields given by a point source at $(9,0): u^{\text {inc }}(x, y)=\log \sqrt{(x-9)^{2}+y^{2}}$. As for the geometries, all of our circular superlenses have $r_{c}=1, r_{s}=4$, which gives a cloaking region $4<r<r_{\#}=8$; our elliptic superlenses, in turn, are constructed by using concentric ellipses of major axes $2 r_{c}$ and $2 r_{s}$ (along the horizontal $x$ axis) and minor axes $r_{c}$ and $r_{s}$, respectively. To quantify the cloaking properties of the superlenses we use the norms

$$
N_{R}(u)=\sqrt{\frac{1}{2 \pi} \int_{0}^{2 \pi}|u(R, \theta)|^{2} d \theta} .
$$

In Table I we display values we obtained for $N_{R}\left(u^{\text {scat }}\right)$ for various values of $R$ and $\delta$ for both the circular and the elliptic superlenses. The data for the circular superlens corroborate previous results ${ }^{2}$ showing that the circular superlens by itself, without additional dielectric bodies $\left(\Gamma_{0}=\varnothing\right)$ or polarizable dipoles $(N=0)$ is perfectly cloaked - that is, in the limit as $\delta \rightarrow 0$ the cylinder is undetectable from the outer field. The data for the elliptic superlenses, in turn, illustrate the fact that superlenses of noncircular cross sections are generally not cloaked. Note that for the circular lens, for a fixed positive value of $\delta$, the magnitude of the potential increases as the boundary of the superlens is approached: the cloaking effect is not uniform in space. It is also worth noting that even for relatively "large" values of $\delta$, the fields scattered by the circular lens are very small.

TABLE I. $N_{R}\left(u^{\text {scat }}\right)$ as a function of $\delta$ for sole circular and elliptical superlenses $\left(\Gamma_{0}=\varnothing\right)$. Notice that the elliptic lens is visible.

\begin{tabular}{ccccc}
\hline \hline & \multicolumn{2}{c}{ Circular lens } & \multicolumn{2}{c}{ Elliptic lens } \\
\hline$R$ & $\delta=10^{-2}$ & $\delta=10^{-12}$ & $\delta=10^{-2}$ & $\delta=10^{-12}$ \\
\hline 6 & $6.24 \times 10^{-4}$ & $2.14 \times 10^{-13}$ & $3.04 \times 10^{-1}$ & $3.15 \times 10^{-1}$ \\
15 & $5.35 \times 10^{-5}$ & $2.52 \times 10^{-21}$ & $1.11 \times 10^{-1}$ & $1.16 \times 10^{-1}$ \\
\hline \hline
\end{tabular}

Table II shows values we obtained of $N_{15}\left(u_{1}^{\text {scat }}-u_{2}^{\text {scat }}\right)$, where $u_{1}^{\text {scat }}$ is the field scattered by the superlens alone, while $u_{2}^{\text {scat }}$ is the field scattered by the superlens and a polarizable dipole of polarizability $\alpha=2$ placed at a distance $d$ above the shell along the vertical axis. The data for the circular superlens verify a previous conclusion from Ref. 4: isolated dipoles are perfectly cloaked when placed inside the cloaking region $r_{s}<r<r_{\#}$ of a circular superlens. The data in Table II corresponding to the elliptic lens, on the other hand, are a significant new result: even though it is not itself cloaked, the elliptic lens does cloak a polarizable dipole.

In our subsequent test cases we study cloaking of dielectric scatterers. In these examples we use dielectrics of dielectric constants $\varepsilon_{0}=4$ whose boundaries $\Gamma_{0}$ are circular of various radii $r_{0}$; we have verified, however, that other values of the dielectric constant and dielectric cylinders of other cross sections give rise to similar results. We first conducted the experiment using the circular superlens, and with the dielectric body centered at two different positions, $(0,6)$ and $(0,9)$ —inside and outside the cloaking region, respectivelyand for various values of $\delta$. The results are shown in Fig. 2: as the size $r_{0}$ decreases, the scattered field decreases quadratically (as expected) when the dielectric is outside the cloaking region, but much faster than quadratically (in fact, quartically in the initial fast-decay portion of the curves around $r_{0}=1$ ) if the scatterer is inside the cloaking region. As claimed earlier, there is a significant cloaking effect, al-

TABLE II. $N_{15}\left(u_{1}^{\text {scat }}-u_{2}^{\text {scat }}\right)$ for configurations including a polarizable dipole a distance $d$ above the shell. When the dipole is close to the lenses cloaking occurs in both the circular and the elliptic cases.

\begin{tabular}{lcccc}
\hline \hline & \multicolumn{2}{c}{ Circular lens } & \multicolumn{2}{c}{ Elliptic lens } \\
\hline$d$ & $\delta=10^{-2}$ & $\delta=10^{-12}$ & $\delta=10^{-2}$ & $\delta=10^{-12}$ \\
\hline 5 & $9.28 \times 10^{-3}$ & $9.25 \times 10^{-3}$ & $1.23 \times 10^{-2}$ & $1.23 \times 10^{-2}$ \\
1 & $7.51 \times 10^{-4}$ & $1.05 \times 10^{-8}$ & $3.62 \times 10^{-4}$ & $1.6 \times 10^{-4}$ \\
0.1 & $-6.27 \times 10^{-5}$ & $1.53 \times 10^{-11}$ & $7.46 \times 10^{-6}$ & $1.45 \times 10^{-6}$ \\
\hline \hline
\end{tabular}




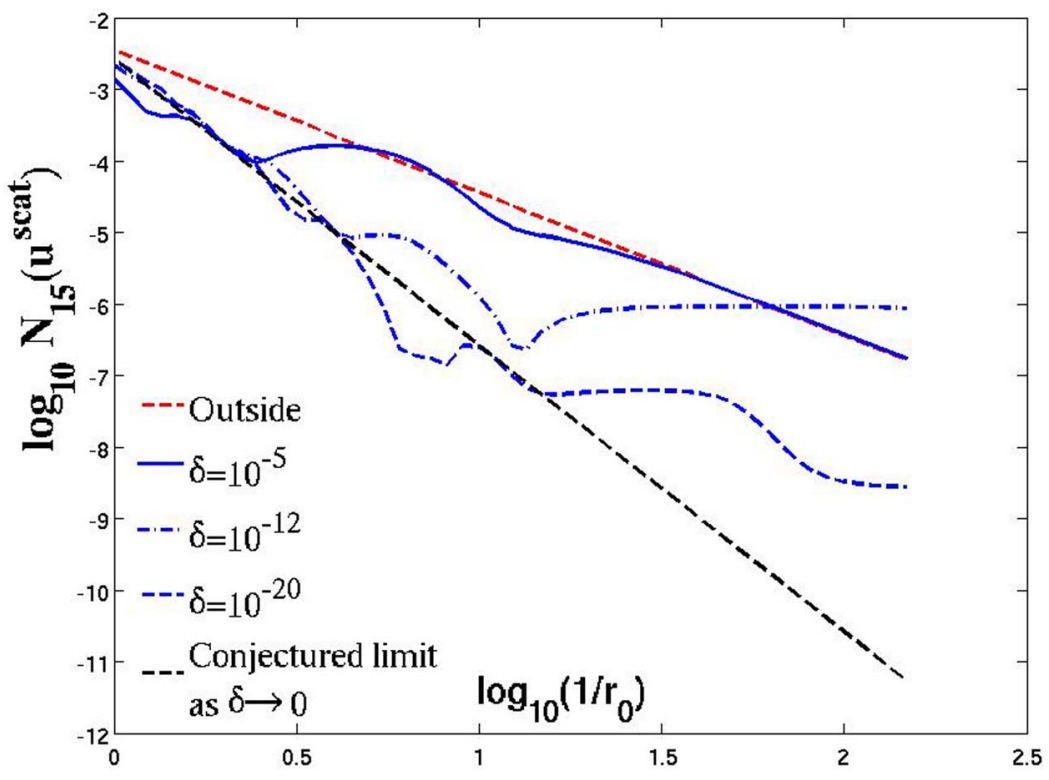

FIG. 3. (Color online) Cloaking of an array of dipoles by a circular superlens as a function of the array size for various values of $\delta$.

though the dielectric is not cloaked perfectly. We note that the cloaking effect is more pronounced as $\delta$ decreases; we conjecture that, for a scatterer placed inside the cloaking region, in the limit as $\delta \rightarrow 0$ the decay in the observed field is exactly quartic as $r_{0}$ decreases to zero. Preliminary numerical experiments, further, indicate that properties of cloaking of small dielectric objects are shared by superlenses of generic cross-sectional curves, see Fig. 1, although, as mentioned earlier, superlenses of generic cross sections are not themselves cloaked. (Compare Table II where related dipolecloaking properties of an elliptic lens were demonstrated.) We note here a different but related remark made in Ref. 4: for $\varepsilon_{c} \neq 1$, dielectric coated cylinders of circular crosssection cloak polarizable dipoles, although for such values of $\varepsilon_{c}$ the coated cylinders are not themselves cloaked.

Since a dielectric body can be viewed as a continuum of polarizable dipoles, and can under normal circumstances be approximated well by a finite collection of them, an expectation ${ }^{4}$ that dielectric scatterers might be cloaked results, reasonably, from consideration of the fact (established in Ref. 4 and confirmed by the numerical results discussed above) that the cylindrical superlens cloaks finite collections of dipoles placed within its cloaking region. Yet, our numerical results indicate beyond doubt that superlenses do not perfectly cloak dielectric bodies: for example, for $r_{0}=1$ the potential of the scattered field shown in Fig. 2 remains of the order of $10^{-1}$ for values of the absorption as small as $\delta$ $=10^{-20}$.

These two apparently contradicting observations can be reconciled by reviewing the proof given in Ref. 4 (Sec. 3) on cloaking for an arbitrary number of dipoles. Indeed, this proof shows that the dipole moment $\mathbf{P}$ of one of a number of polarizable dipoles contained in the cloaking region [located, say, at position $z=(x, y)$, at a distance $r=|z|$ from the origin] is bounded by $B_{C}(\delta)=C \delta^{\log \left(r_{\#} / r\right) / \log \left(r_{s}^{2} / r_{c}^{2}\right)} / \sqrt{\log (\delta)}$, where $C$ is a constant, related to a certain Vandermonde matrix that depends on the position of all the polarizable dipoles within the cloaking region. This bound shows that any finite and fixed distribution of polarizable dipoles located within the cloak- ing region is cloaked. It can be checked, however, that the constant $C$ tends to infinity very rapidly as the distance between dipoles tends to zero: if all $n$ dipoles are within distance of order $\eta$ of each other, for example, then, as $\eta$ tends to zero, $C$ tends to infinity at least as fast as $\eta^{1-n}$. This fact, coupled with the slow decay of $B_{C}(\delta)$ as $\delta \rightarrow 0$ require that the cloaking result of Ref. 4 be qualified: a fixed or otherwise well separated distribution of finitely many polarizable dipoles within the cloaking region is cloaked. The result of Ref. 4 does not apply if no restriction is placed on the distances between dipoles. Since dielectrics are closely packed arrays of microscopic dipoles, a failure of the cloaking property to hold for such materials is understandable: the cloaking result ${ }^{4}$ does not apply to dielectrics.

We illustrate this discussion with the results of some numerical experiments in which we study the response that arises as an array of dipoles is placed within the cloaking region of the circular superlens. The array consists of those dipoles in an evenly spaced set of $16 \times 16$ dipoles inside a square of size $2 r_{0}$ that lie inside the circumscribed circle of radius $r_{0}$. If $r_{0}$ is the radius of the circular dielectric scatterer studied earlier, the polarizability $\alpha_{d}$ of the dipoles is chosen in such a way that, in absence of the coated cylinder, the response of the dipole array to an outside field is approximately equal to that of the dielectric scatterer. (The polarizability $\alpha_{d}$ was tuned once for $r_{0}=1$, and, in accordance with the Clausius-Mossotti formula, for decreasing values of $r_{0}$, $\alpha_{d}$ was renormalized with respect to the cross-sectional area: $\alpha_{d}\left(r_{0}\right)=\alpha_{d}(1) r_{0}^{2}$. Note from Figs. 2 and 3 that, when placed outside the cloaking region, for example, the dipole array gives rise to a scattered field very close to that resulting from the dielectric scatterer.) For various values of $r_{0}$, we evaluated the scattered fields that resulted as the dipole array was first placed inside and then placed outside of the cloaking region. The results of these experiments are shown is shown in Fig. 3: clearly, in both cases, the response of the lens/ dipole-array configuration is similar to that of the dielectric/ lens configuration. 
Using numerical solvers of high precision we have thus demonstrated that both circular and noncircular superlenses cloak dielectric scatterers to a significant degree: the scattered field induced by a dielectric scatterer placed inside the cloaking region decreases significantly faster, as the scatterer size is reduced, than the field induced by a scatterer placed outside the cloaking region. This effect is nonuniform, depending on the distance to the lens, the relative ratio of the lens' radii, and the loss $\delta$ in the negative index material.
The authors gratefully acknowledge support from USAFOSR (FA9550-05-1-0006) and NSF (DMS-0408040).

${ }^{1}$ J. B. Pendry and D. R. Smith, Phys. Today 57, 37 (2004).

${ }^{2}$ N. A. Nicorovici, R. C. McPhedran, and G. W. Milton, Phys. Rev. B 49, 8479 (1994).

${ }^{3}$ J. B. Pendry, D. Schurig, and D. R. Smith, Science 312, 1780 (2006).

${ }^{4}$ G. W. Milton and N. A. Nicorovici, Proc. R. Soc. London, Ser. A 462, 3027 (2006).

${ }^{5}$ R. Kress, Linear Integral Equations, 2nd ed. (Springer, New York, 2003). ${ }^{6} \mathrm{See} \mathrm{http://crd.lbl.gov/} \mathrm{dhbailey/mpdist/mpdist.html.}$ 